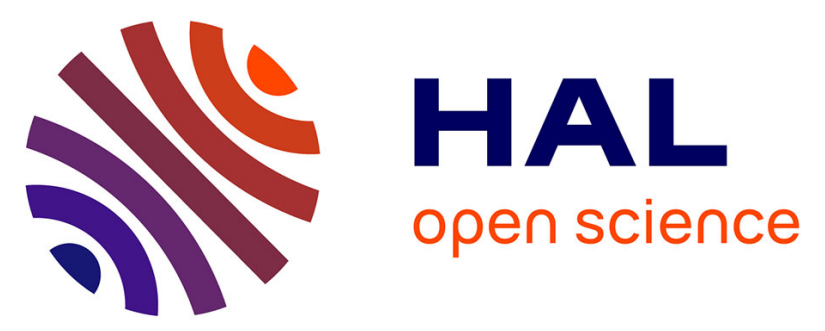

\title{
Emergent Complex Behaviors for Swarm robotic Systems by Local Rules
}

Abdelhak Chatty, Ilhem Kallel, Philippe Gaussier, Adel M.Alimi

\section{To cite this version:}

Abdelhak Chatty, Ilhem Kallel, Philippe Gaussier, Adel M.Alimi. Emergent Complex Behaviors for Swarm robotic Systems by Local Rules. IEEE Symposium on Computational Intelligence on Robotic Intelligence In Informationally Structured Space (RiiSS), Apr 2011, France. pp.69 -76. hal-00955949

\section{HAL Id: hal-00955949 \\ https://hal.science/hal-00955949}

Submitted on 5 Mar 2014

HAL is a multi-disciplinary open access archive for the deposit and dissemination of scientific research documents, whether they are published or not. The documents may come from teaching and research institutions in France or abroad, or from public or private research centers.
L'archive ouverte pluridisciplinaire HAL, est destinée au dépôt et à la diffusion de documents scientifiques de niveau recherche, publiés ou non, émanant des établissements d'enseignement et de recherche français ou étrangers, des laboratoires publics ou privés. 


\title{
Emergent Complex Behaviors for Swarm Robotic Systems by Local Rules
}

\author{
Abdelhak Chatty ${ }^{1,2}$,Ilhem Kallel ${ }^{1}$,Philippe Gaussier ${ }^{2}$ and Adel M. Alimi ${ }^{1}$ \\ ${ }^{1}$ REGIM: REsearch Group on Intelligent Machine \\ University of Sfax, National School of Engineers (ENIS) \\ Sfax, Tunisia \\ ${ }^{2}$ ETIS: Neuro-cybernetic team, Image and signal Processing \\ Cergy-Pontoise University \\ Paris, France \\ \{abdelhak_chatty, ilhem.kallel, adel.alimi\}@ieee.org, Gaussier@ensea.fr
}

\begin{abstract}
This paper describes a clustering process taking inspiration from the cemetery organization of ants. The goal of this paper is to show the importance of the local interactions which allow to produces complex and emergent behaviors in the field of swarm robotics. A series of simulations enables us to discuss and validate the fact that the changes of rules allow generating other emergent behaviors for the achievement of tasks.
\end{abstract}

Keywords-component; Emergent bihaviors; taking-deposit rule; local rules; clustering; swarm robotics.

\section{INTRODUCTION}

The behaviors exhibited by ants are known to be the most mature among swarm intelligence techniques. Indeed, the observation of ant colonies led to so called ant colony algorithms [1]. These algorithms revolve around a key concept called stigmergy which is an indirect coordination activity in an unknown environment: Ants are attracted to each other by a chemical material called pheromone. The Ant algorithms provide powerful methods for the design of algorithms and optimization of distributed problems involving a collaborative swarm behavior [2], [3], [4], [5], [6]. It seems that in an unknown environment, one of the most important problems related to multi-robots systems is to decide how to coordinate actions in order to achieve tasks in an optimal way [7]. It is also very important to know what needs to be accomplished and the number of robots required for the task. In addition to this, it is important to know how to make the multi-robot systems self-organized.

It is clear that execution of a cargo pushing task for example is more natural if it is accomplished by a multi-robot system. Though the introduction of ant algorithms, based on the modification of environment used as a minimal communication tool into the world of robotics, improves the communication quality between robots. We start really to speak about swarm robotics[7], [8], [9], [10], [11], [12], [13]. In fact, when an ant tries to move a cargo (e.g., food) alone, it spends moments to test the resistance of the cargo to forces applied by varying the orientation of the ants body.Changing the direction of the applied strength can be enough to result in actual movement of the cargo. Should the realignment be inadequate, the ant releases the cargo and tries another position or direction from which to seize and move the cargo.If multiple adjustment attempts are not successful, the ant recruits other ants [7]. In the same area Kazuhiro Ohkura present in [14] an evolutionary robotics approach, in which robot controllers are designed by evolving artificial neural networks. Many works such as those cited by Kube and Zhang [9] employ ant algorithms to solve object transportation problems.

Other research as consist of changing the ant algorithm using a new technique for multi-robot pheromone placement. This technique enables robots to place more pheromones in the tasks that are about to be fulfilled: A very large amount of pheromone is placed on the cargo items that are close to successfully being moved or close to reaching their destinations, hence requiring a great force to move them. A small amount of pheromone is otherwise placed on the cargo. This strategy helps to attract additional robots to complete the task when needed [11]. In fact, the amount of pheromone is constant whereas in [12], [13] it is variable and depends on task difficulty and on the robots strength. Dasgupta describe also in [15] an emergent algorithm inspired by stigmergy in insect colonies, to solve the n-collaborative pursuit-evasion game. The problem of task allocation in the field of cooperative robotics is addressed by several studies especially in the context of unknown environments. Each robot has to adapt to its environment without any training stage. Moreover, the materialization of pheromone into real robots remains problematic in spite of the various attempts [16], [17].Lets consider another view of swarm intelligence inspired from ant colony optimization called Counter-Ant Algorithm (CAA) [18], [19]. The robots collaborative behavior is based on repulsion instead of attraction to pheromone that represents the core of ants cooperation. The robots reaction consists, henceforth, of avoiding paths covered by this chemical substance. The modified version includes a solution for stagnation recovery using pheromone positions. In recent years, research has been oriented more towards 
the development of real-time systems and particularly the study of hybrid methods to ensure scalability of behavior with respect to the dynamic nature of the environment, in [20] the authors present a fuzzy system for avoiding the collaboration stagnation and to improve the counter-ant algorithm . The robots collaborative behavior is based on a hybrid approach combining the CAA and a fuzzy system learned by MAGAD-BFS (Multi-agent Genetic Algorithm for the Design of Beta Fuzzy System).

To improve the uses of swarm robotics, we show in this paper how we can generate an emergent behavior allowing the realization of different tasks. In particular, our work deals with the problem of controlling a colony of agents moving objects. Indeed, we begin to study a basic level where the capacity of agents is limited to a level more complex achieved by the use of cognitive agents. The remainder of this paper is organized as follows. Section II overviews the use of emergent proprieties. Sections III and IV present and discuss the proposal: the local rules to achieve the clustering of objects and the clustering of resources. Finally, the last section concludes the paper.

\section{THE USE OF EMERGENT PROPRIETIES}

Emergent behavior is one of the main topics of research in the field of swarm robotics. The stigmergy is the factor that allows the creation of many emergent structures through the collaboration between robots. Indeed, interaction between agents needs not to be complex to produce global emergent behavior. We need just the use of a limited vision and a simple local interaction without the need of the capacity for spatial orientation or memory; the robots are able to achieve tasks and to create an emergent behavior. Holland presents in [10]the operation of stigmergy and the self-organization. Mataric in [21] describes the way to understand the types of simple local interactions which produce complex group behaviors. So we tried to understand the types of simple local interaction allowing the process of organization of cemetery of ants.

\section{A. The organization of ants' cemetery}

The phenomenon that is observed in ants' cemetery is the aggregation of dead bodies by workers. If dead bodies are randomly distributed in space at the beginning of the experiment, the workers will form clusters within a few hours. The basic mechanism underlying this type of aggregation phenomenon is an attraction between dead bodies mediated by the ant workers: small clusters of dead bodies grow by attracting workers to deposit more items. It is this positive feedback that leads to the formation of larger and larger clusters. In this case it is therefore the distribution of the clusters in the environment that plays the role of stigmergic variable [2]. If we observe on closer, we see that ants behavior is to amplify local deposits: more there was a dead body in a cluster more the probability of deposits is important. Moreover, more the cluster is voluminous more the probability of taken is low. For taken, ant will want to take a dead body when the cluster is small, because its easy to take it. For deposit, ant will want to deposits the dead body when the cluster is large [22].

\section{B. Related works}

By exploiting the self-organized clustering of objects in a closed arena taking inspiration from the cemetery organization of ants, researchers have been able to design a number of successful algorithms: Deneubourg in [4] have proposed a model relying on biologically plausible assumptions to account for the phenomenon of dead body clustering in ants. Gaussier [23] show that acting on objects simplifies the reasoning needed by a robot and allows clustering of scattered objects. The robots are programmed to move randomly and to take an object if they find one and to deposit it if they find another object. The result shows that the heaps built are linear and long, and this is due to an interpretation error: tow objects together can be perceived as a wall and the robots then avoids them. So the operation depends on the robot orientation with regard to the heap. Becker et Holland [24], [10]studied the clustering and sorting of colored frisbees by a group of real robots. Martinoli [25] studied the clustering of small cylinders by a group Khepera robots, the rules are based on the perception sensors: if all the four sensors of Khepera are very active, this indicates that there is a large obstacle in front of it and has to avoid it. And stays in this mode until none of the proximity sensors notice any obstacle. Now if the two lateral proximity sensors are not saturated, it indicates to Khepera that there is a small object in front of it. Lan in [26] presents a multi-robot system for collective clustering task. It consists of homogenous robots with only local sensing ability and four simple reactive behaviors. M.Martin [27] shows that the observed clustering of corpses can be explained by a statistical effect. So we will try to use the same phenomenon for dead bodies but we will change the locale rules to achieve an emergent behavior allows the clustering of objects.

\section{OBJECTS CLUSTERING BASED ON LOCAL DISTANCES}

The phenomenon observed in ants cemetery represents our inspiration source for realizing the clustering of objects by agents. The behavior of agent in the environment is very simple; it allows the search of objects either for taking or for deposit. The agents move in the environment randomly looking for objects. When agent finds them in her proximity, it chooses the nearest object for transport when it is on mode taking. If it is on mode deposit, the agent chooses the distant object to deposit inside. It should be noted that agents are equipped by a limited field of vision which allows them to detect objects for participate in operation of transport, and to avoid the other agents.

\section{A. The local taking-deposit rules}

The mode of taking is based on the fact to choose the nearest object based on (1): In the instant of detection of objects, each agent calculates the minimum distance $\mathrm{D}$ between its position $\mathrm{Pi}$ and the position of the objects $\mathrm{Oj}$ detected in the field of vision. 


$$
D_{(\text {Taking })}=\min (d(P i, O j))
$$

An agent starts on deposit mode, and tries to look for the distant object to deposit to beside. As seen in (2), for each transported object, the agent calculates the maximum distance $\mathrm{D}$ between its position $\mathrm{Pi}$, and the position of other objects $\mathrm{Oj}$ detected in the field of vision.

$$
D_{(\text {Deposit })}=\max (d(P i, O j))
$$

With $\mathrm{d}$ is the Euclidian distance of the environment.

\section{B. StagnationRecoveryusing the Same Rules}

By analyzing the behavior of agents, we note a stagnation situation [28]: when agent accomplishs an operation of deposit in an area containing only one other object, it enter in infinite operations of taking-deposits the same objects. However, with the repeated use of the same local taking-deposit rules, agent arrives to make transition into the environment, and thus move by varying the positions of objects. With the phenomenon of emergent transition the probability to find other objects is big, what allows the agent to take and deposit other objects. The Fig. 1 presents the flowchart of the collaborative transportation task based on simple rules.

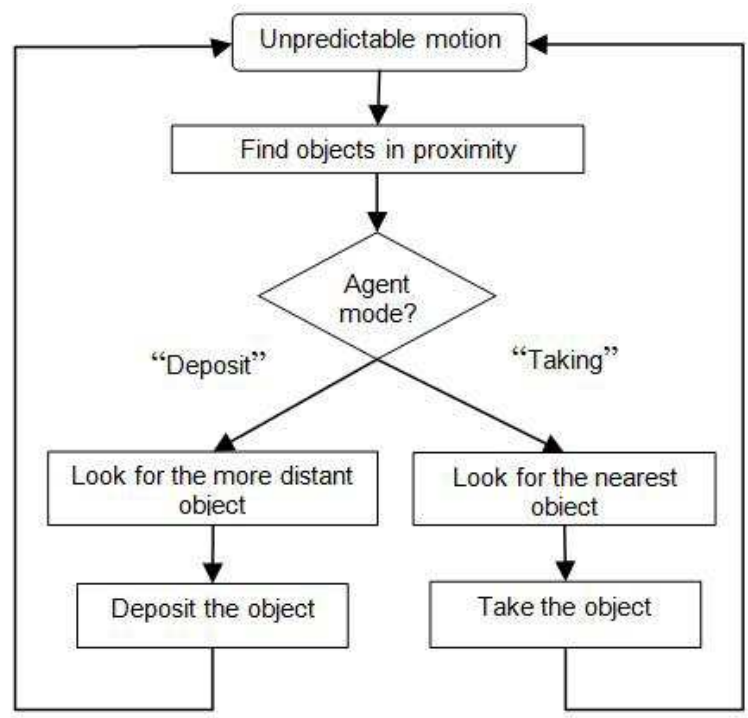

Fig. 1. Collaborative transportation task based on simple rules

\section{Simulation}

Collaboration between robots is done in a completely unknown environment. It is made up mainly by objects and robots which are implemented and generated by the simulation platform Madkit. These robots are provided by a field of vision enabling them to detect the objects. Fig. 2a presents a scenario of our simulation environment at the starting time $\mathrm{t}=0$, containing two robots with a field of vision equal $80 \mathrm{~cm}$ to strengthen the use of locals rules, and 85 objects. The agents start to move randomly in the environment using the rules of taking and deposit. In Fig.2b and Fig.2c we note that the global behavior of our system emerges to create a large number of clusters scattered in the environment. These clusters are builded through the collaboration of agents across the stigmergy embodied in the objects into the environment. Fig.2d and Fig.2e show that the number of clusters continues to decrease and their density increases. Finally, Fig.2f shows that the emergent behavior of our system based on simple locals rules allows to reduce the number of clusters and move them with an emergent and collaborative manner to create a single cluster. As Dorigo says in [2] that if the experimental arena is not sufficiently large, or if it contains spatial heterogeneities, the clusters will be formed along the borders of the arena, so we note that the cluster is formed along the border of our environment.

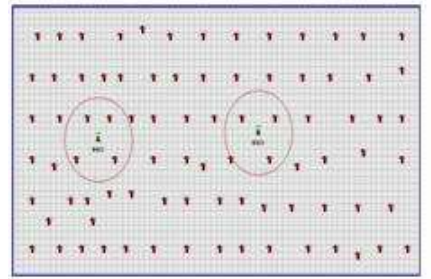

(a) $\mathrm{t}=0 \mathrm{~s}$

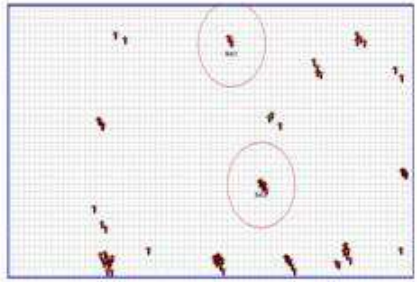

(c) $t=235 \mathrm{~s}$

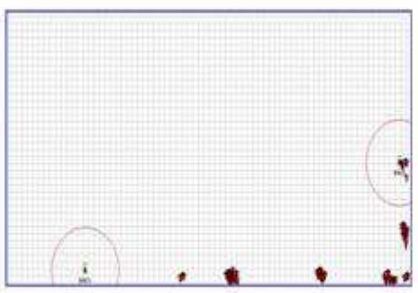

(e) $t=653 \mathrm{~s}$

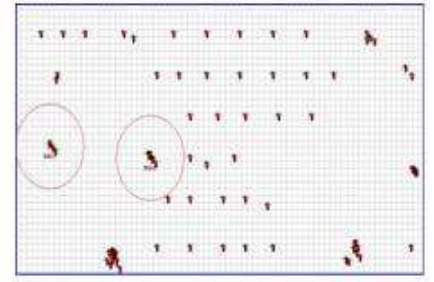

(b) $t=120 \mathrm{~s}$

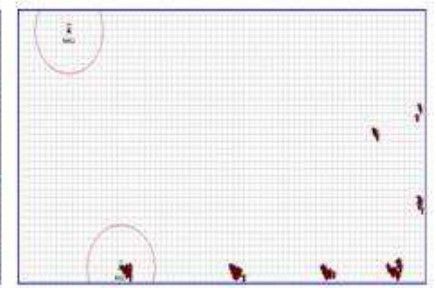

(d) $t=487 \mathrm{~s}$

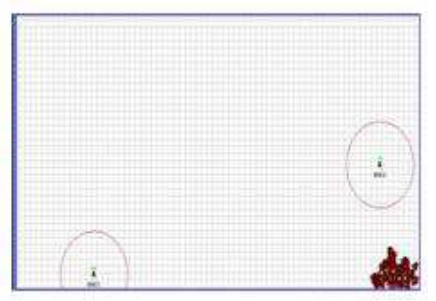

(f) $t=827 \mathrm{~s}$
Fig. 2. Creation of emerging clusters

\section{The physical emerging phenomenon}

It is noted from the Fig. 3, that the rate of occupancy of 85 objects in the environment is equal $2.95 \%$. Thus, our system was able to reduce this rate with the creation of clusters of objects: the rate goes up by $2,04 \%$ until it reaches $0,69 \%$ where we have one cluster of objects. So we reduce 4 times the rate of occupancy of objects. Fig.3, shows all the rate of occupancy over the time. In other side, the use of two robots allowed us to vary the number of clusters from 19 to 1 in the same environment. The curve 4 , shows the change in cluster size over time. From 60 to 235 steps of time, we notice the temporary creation of small clusters whose size varies between 
0 and 10 , which end to disappear. The disappear is due to the transition phenomenon: the destruction of certain clusters can sustain or create other in the environment. From 235 until 487 steps of time we can see the creation of large clusters whose size varies between 10 and 20. After 653 steps of time we see the destruction of the majority clusters and finally 827 step of time, we see the destruction of all the clusters and the construction of a single cluster that brings together all the objects in the environment. We can say that the transition phenomenon allows to optimize the rate of occupancy of objects and to create just one cluster in the arena.

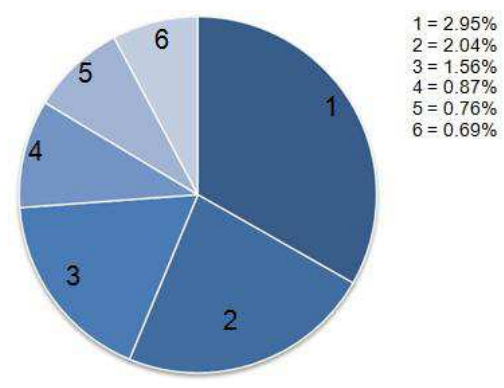

Fig. 3. The rate of occupancy of objects over the time

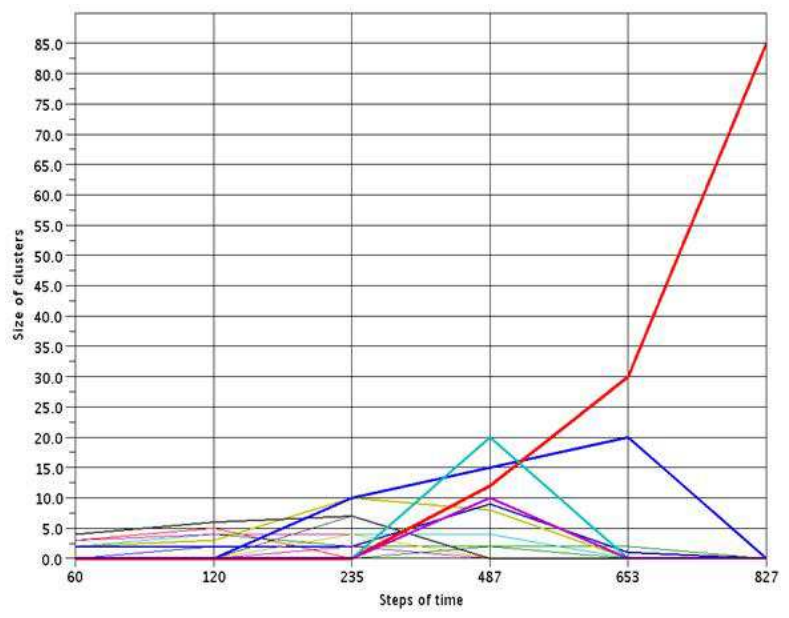

Fig. 4. The evolution of the clusters size

\section{E. The temporal emerging phenomenon}

Fig. 5, shows (from 1 to 2 robots) that in spite of the fixed field of vision, the convergence time is improved, from 1722s with only one robot to $827 \mathrm{~s}$ with two robots. This emphasizes the effectiveness of swarm robotics in the collaboration works. With 2 to 6 robots the convergence time continues to decrease to $553 \mathrm{~s}$, which shows that increasing the number of robots strengthens the emergent behavior of the creation of clusters and accelerates the process. However with more than 6 robots, the convergence time increases, thats confirms that the uses of the local rules allows to give in all cases an emergents proprieties independently of the number of robots but that will affect the convergence time. The question that arises is to know why the convergence time increased when we added the robots while the goal was to strengthen the emerging behavior! When we observe the simulation we noticed that there is another ermerging behavior which was created: its the destructive behavior. Its a purely emergent: A number of agents in the taking mode can frequently be met next to a cluster, so instead of strengthening it by filing of other objects, the agents will take all the objects of the cluster which leads to its destruction. This emergent phenomenon will slow down the convergence time of our system because that slows down also the creation of clusters. The curve 6 , shows the number of clusters over time. We note that the number and size of clusters increases with increasing number of robots which increases also the convergence time of our system. Indeed, the transition of large clusters with the use of 9 robots will take a long time compared to clusters whose size is less than 20 (with two robots).

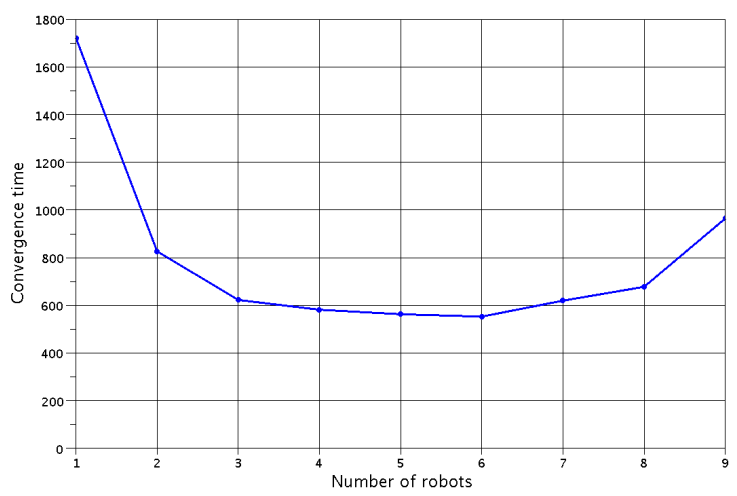

Fig. 5. The evolution of the convergence time

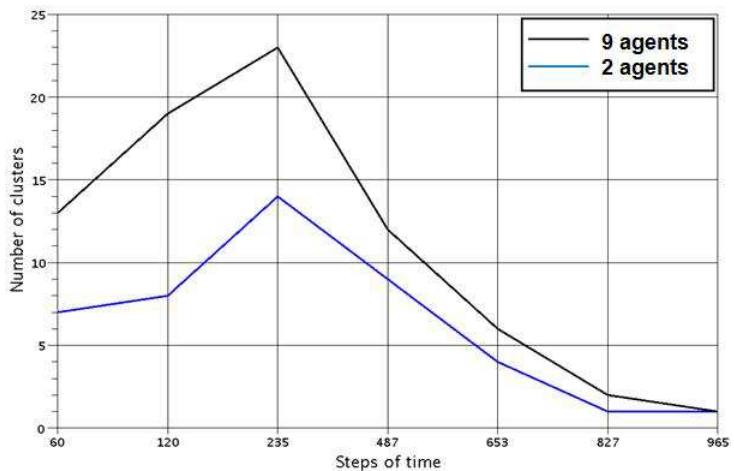

Fig. 6. Evolution of the number of clusters

\section{THE EMERGING CLUSTERING RESOURCES}

We have study a basic level where, the capacity of agents is limited and we have shown the effectiveness of local rules to generate emergent behaviors allowing performing the task. If we continue to exploit the phenomenon of dead body by varying the taking-deposit rules in a more complex level achieved by the use of cognitive agents, to solve an optimization problem called resources transportation. 


\section{A. The problematic situation}

In the simulation environment, we suppose three initial types of sources: water, food and nest, wich are scattered. We seek in this part, to solve the transportation problem: This means of taking resources and put them in relevant places to optimize the planning agents to search resources, to achieve the survival of other agents, and to have a dynamic stable system. We use three motivations for agents (eating, drinking, and resting). Each one associated with a satisfaction level that decreases over time and increases when the agent is on the proper source. When a level of satisfaction falls bellows a given threshold, the corresponding motivation is trigged so that the robot has to reach a place allowing to satisfy this need [29].

\section{B. The internal architecture of agents}

The work of OKeefe [30] shows a special cells in the rats hippocampus that fire when the animal is at a precise location. These neurons have been called place cells. We do not directly use them to plan or construct a map, we rather use neurons called transition cells. During exploration these transition cells are created and allow to learn cognitive map [31]. The cognitive map allows to avoid planning problem when trying to use the map before a complete exploration of the environment has been reached and to allow also a single merging of action between sensor-motor and the planning strategies. The cognitive map acts as internalization of pheromone traces coupled with an imitative behavior, it allows the same results without the need to leave a physical trace in the environment. At the individual level, agents can rely on an on-line continuous building of a cognitive map whose structure depends on their own experience and discovery of the environment in which they live. The cognitive map aims at making agents learn the news positions of resources and evolve in a dynamically changing environment, where some sources can disappear when intensively visited for a long time and other can appear somewhere in the environment.

\section{The local taking-deposit rules}

To keep a fixed number of resources deposited in relevant places we try to use local rules. The condition of taking computed by (3) is as follows: The probability that agent want to take increase when he realizes that a source is abandoned by other agents. So, more agents are near a source, more the probability of taking decreases and vice versa.

$$
\operatorname{Pr}_{(\text {Taking })}=e^{-(N R N+\operatorname{var} 1)}
$$

Equation (4) presents condition of deposits as follows: The agent has want more to deposit, if he realizes that the place is frequented by other agents and it is far on the original source where he conducted the transaction of taking, this ensures the relevance of the location of resources. The deposit operation is built on the concept of reinforcement: the agent puts in the resources that already exist

$$
\operatorname{Pr}_{(\text {Deposit })}=\left(1-e^{-N R N / \operatorname{var} 2}\right) *\left(1-e^{-t / \operatorname{var} 3}\right)
$$

Where NRN is number of robots in the neighbor, $t$ is the time from the taking and var1,var2 and var3 are numbers which makes it possible to control the rules.

\section{The preliminary results of the global behavior}

To better analyze the behavior of our system, we run simulations by varying the initial number of agents in the environment, then we obtain the following results. It is noted that var $1=1$,var $2=2$ and var $3=1000$. Fig. 7 and Fig. 8 show that the number of persistent taking and of deposits increases with the increase of the number of agents in the environment. Fig.9 shows also that agents try to strengthen the existing ressources. The number of reinforcments increases with the increases of the number of agents in the environment. This limits the number of deletions ressources and allows to maintain a fixed number resources in the environment. Indeed, each resource has its own life, and once it reaches 0 the resource disappears (see Fig.10). Fig.11 shows that the average of convergence time also increases with increasing the number of agents to reach 30326 time steps, which is considered as high. In Fig. 8 , the number of persistent deposits varies between 3 and 13, also the Fig.11 shows that the average of convergence time varies between 8737 and 30326 steps time.

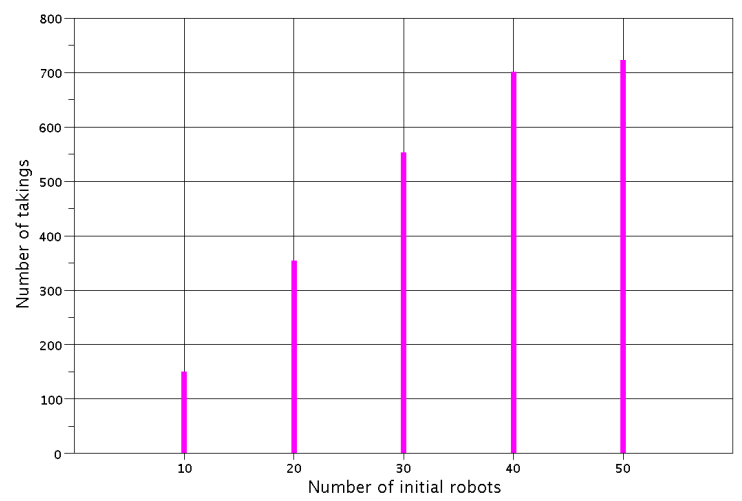

Fig. 7. Influence of the number of robots on the number of taking

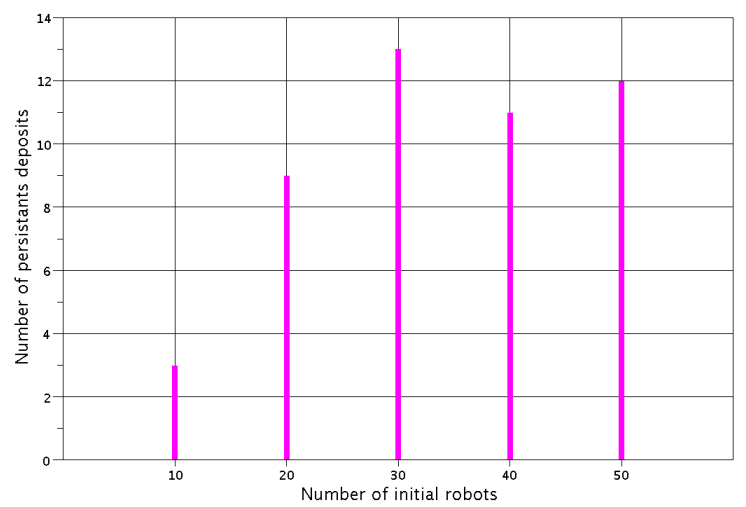

Fig. 8. Influence of the number of robots on the number of persistants deposits 


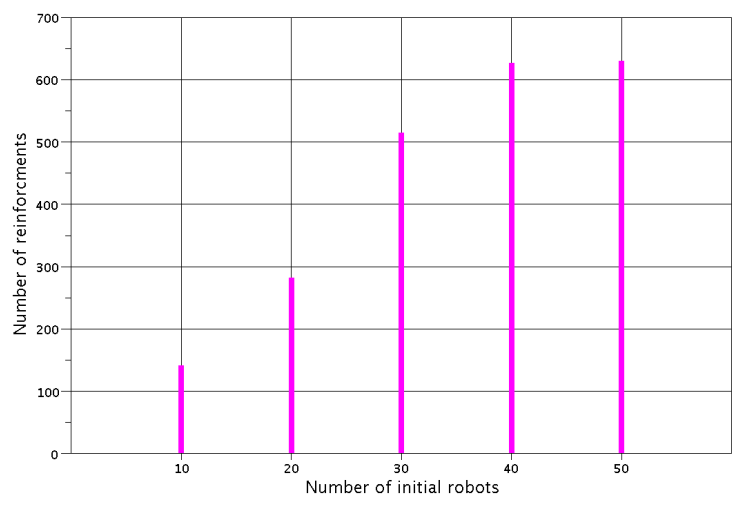

Fig. 9. Influence of the number of robots on the number of reinforcements

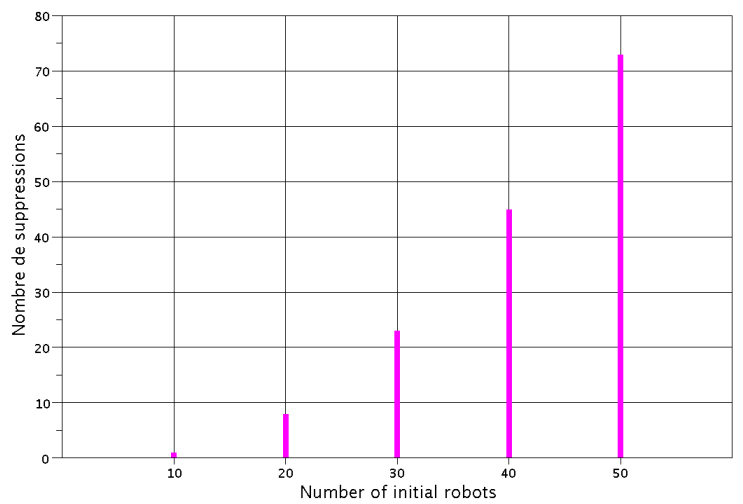

Fig. 10. Influence of the number of robots on the number of suppressions

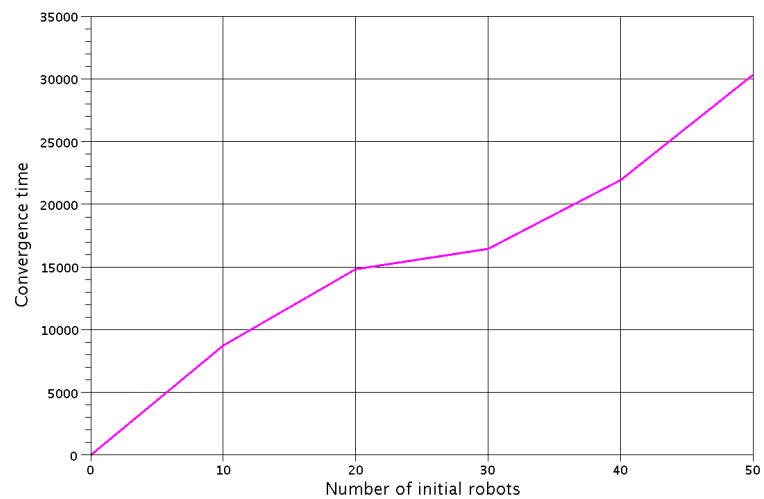

Fig. 11. Influence of the number of robots on convergence time

\section{E. Emergence improvement by refinement rules}

To improve performance by controlling the number of persistent deposition and by limiting the convergence time we try to refine the local rules. We integrate in deposit rule, two environmentals factors $\alpha$ et $\beta$ expressed by (5).

$$
\operatorname{Pr}_{(\text {Deposit })}=\left(1-e^{\alpha(-N R N / \text { var } 2)}\right) *\left(\left(1-e^{\beta(-t / \text { var } 3)}\right)\right)
$$

By varying the values of $\alpha$ and $\beta$, we notice that increasing the values of environmental factors increases the convergence time and the number of persistent deposits and vice versa. To focus on changes in the number of persistent deposits and the convergence time of our system over time, we perform different simulations with $\alpha=\beta=0.3$ when variying the number of agents.

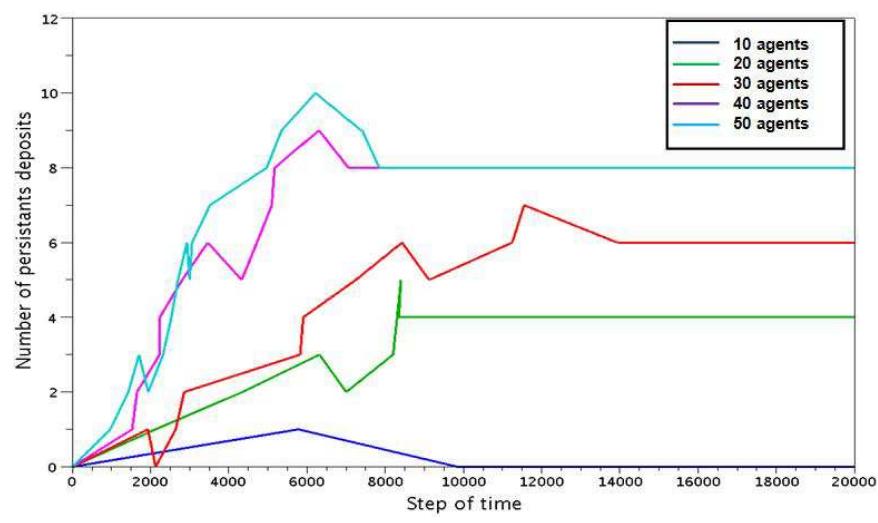

Fig. 12. The average of persistent deposit and convergence time

Fig. 12 shows that the refinement rules allows us to control the convergence time and the number of persistent deposits. We also note that until 4500 steps of time, the number of persistent deposits is not stable. From 6000 steps of time, the number of deletions decrease that's allows increases the number of reinforcements. For 10 agents, the number of persistent deposits $=0$, this shows that the cooperation between agents is not succeeded in order to locate relevant locations in the environment. The average time of convergence for 40 and 50 agents in the area is equal 7000 step of time. For 10 and 20 agents it's equal to 9000 steps of time. However, it's 13000 steps of time for 30 agents. We also note that increasing number of agents from 40 to 50 does not change the number of persistent deposits. We can then say that the number of persistent deposits does not depend on the number of agents in the environment, and the agents just make the reinforcements of the emergent behavior.

\section{F. The emergent Behavior of agents}

Fig. 13a presents the simulation environment at $\mathrm{t}=0$ where the original sources are scattered. The Agents move randomly in the environment with a taking mode, with a field of perception that can minimize the ability of agents to perceive the environment. When passing of the resources (nest, food or water) agent increases its level of satisfaction and executes the locale rule of taking, to transporting a quantity of this resource: the probability of take increases when the agent does not detect other agents next to the source. If an agent transports successfully, it continues its travel but in the deposit mode: the probability of deposits increases more when agent detects other agents and it moves away from the source. Once deposited, the new resources allow to agents to increase their satisfactions level (see Fig.13b, Fig.13c and Fig.13d). If the agent passes through a resource deposited and it carries a similar resource it reinforces it, otherwise it increases its level of satisfaction and decreases the amount of the resource. Each resource has its own life once it reaches 0 the resource 
disappears. If we consider (Fig.13c and Fig.13d) or (Fig.13f and Fig.13e), we notice the disappearance of resources.

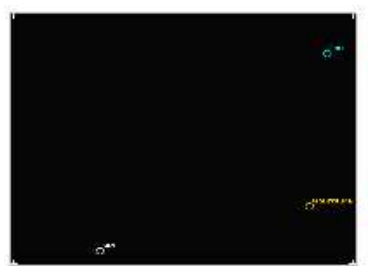

(a) $\mathrm{t}=0 \mathrm{~s}$

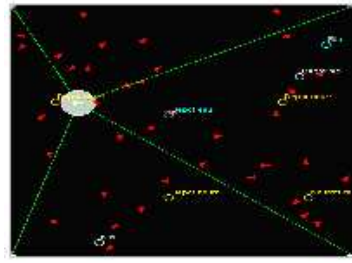

(c) $\mathrm{t}=3628 \mathrm{~s}$

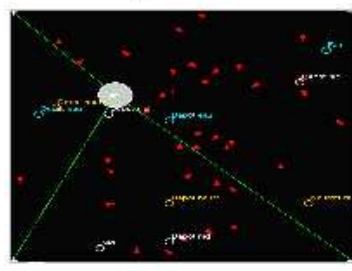

(e) $\mathrm{t}=7741 \mathrm{~s}$

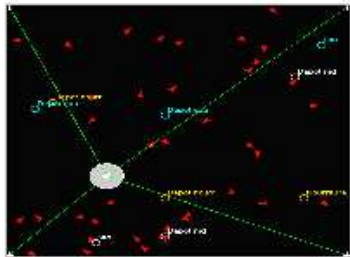

(g) $\mathrm{t}=20000 \mathrm{~s}$

Fig. 13. Emergent transportation of resources

The behavior of agents based on local rules allows the creation of emerging resources in relevant places frequented by the population of agents. The same behavior also allowed to keep a fixed number of resources through the environment and to remove by an emerging way other resources located in not relevant places: hence the stability of the environment (Fig. 13f and Fig. 13g), we note in this case that the number of persistent deposits does not change. The final resources that exist in the environment are the emergence of an individual decision of an agent (when creating the resource) to a collective decision of the entire population (through the maintain of resource via the reinforcement). Fig.13f represents the environment at the stability of our system (at 7932 step of time); we note that the emergent behavior of cognitive agents helped create 6 dispersed clusters allowing the survival of the other and the optimization of planing to find resources. We see also that the clusters are not formed along the borders of the arena. This experiment confirms that acting on objects simplifies the reasoning needed by a robot and allows clustering of scattered objects [23]. The Fig. 14 shows the average number of the agents passages on resources. We see that resources deposited by agents are exploited which proves they are well placed in areas frequented by other agents. The initial sources are poorly operated favoring reinforcement operation.

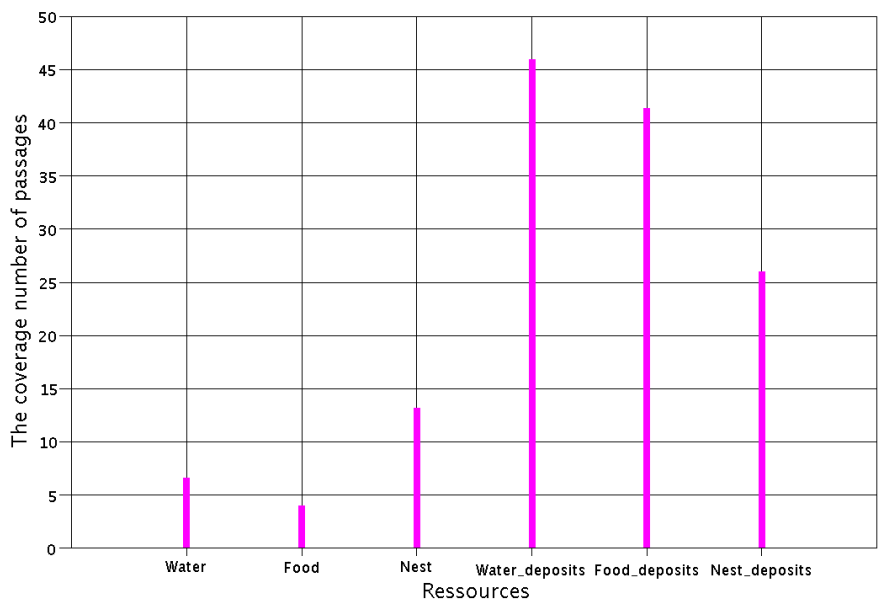

Fig. 14. Number of the agents passages

\section{CONCLUSiON}

This paper describes a clustering process taking inspiration from the cemetery organization of ants. We aim to show how we can generate an emergent behavior based on local rules, allowing the realization of different tasks. Indeed, we studied a basic level where the capacity of agents is limited and we have shown the effectiveness of local rules to generate emergent behaviors that allow performing the task. By varying the taking-deposit rules in a more complex level achieved by the use of cognitive agents, we show that we can solve the optimization problem of resources transportation and we have highlighted the role of the cognitive map to manage the complexity of the environment via recovering emergent behaviors.

\section{ACKNOWLEDGMENT}

Authors thank the financial support of the Tunisian General Direction of Scientific Research and Technological Renovation (DGRSRT), under the ARUB program 01/UR/11 02, and the Institut Frana̧is de Coopration.

\section{REFERENCES}

[1] M. Dorigo and G. D. Di Caro, The Ant Colony Optimization MetaHeuristic. London: McGraw-Hill, 1999, pp. 11-32.

[2] M. Dorigo, E. Bonabeau, and G. Theraulaz, "Ant algorithms and stigmergy," Future Gener. Comput. Syst., vol. 16, no. 9, pp. 851-871, June 2000.

[3] M. Dorigo, M. Birattari, and T. Stutzle, "Ant colony optimization," IEEE Computational Intelligence Magazine, vol. 1, no. 4, pp. 28-39, November 2006.

[4] J. L. Deneubourg, S. Goss, N. Franks, A. S. Franks, C. Detrain, and L. Chrétien, "The dynamics of collective sorting robot-like ants and ant-like robots," in Proceedings of the first international conference on simulation of adaptive behavior on From animals to animats. Cambridge, MA, USA: MIT Press, 1990, pp. 356-363. 
[5] H. Szu, P. Chanyagorn, W. Hwang, M. Paulin, and T. Yamakawa, "Collective and distributive swarm intelligence: evolutional biological survey," International Congress Series, vol. 1269, pp. 46-49, August 2004.

[6] Kurabayashi, Daisuke, Urano, Katsunori, Funato, and Tetsuro, "Emergent transportation networks by considering interactions between agents and their environment," Advanced Robotics, vol. 21, no. 12, pp. 13391349, 2007.

[7] C. Kube and E. Bonabeau, "Cooperative transport by ants and robots," Robotics and Autonomous Systems, vol. 30, no. 1-2, pp. 85-101, January 2000.

[8] G. Dudek, M. Jenkin, E. Milios, and D. Wilkes, "A taxonomy for swarm robots," in Intelligent Robots and Systems '93, IROS '93. Proceedings of the 1993 IEEE/RSJ International Conference on, vol. 1, Jul. 1993, pp. $441-447$ vol.1.

[9] R. C. Kube and H. Zhang, "Collective Robotics: From Social Insects to Robots," Adaptive Behavior, vol. 2, no. 2, pp. 189-218, September 1993.

[10] O. Holland and C. Melhuish, "Stigmergy, self-organization, and sorting in collective robotics," Artif. Life, vol. 5, no. 2, pp. 173-202, 1999.

[11] D. Yingying, H. Yan, and J. Jingping, "Multi-robot cooperation method based on the ant algorithm," in Swarm Intelligence Symposium, 2003. SIS '03. Proceedings of the 2003 IEEE, 2003, pp. $14-18$.

[12] E. Borzello and L. Merkle, "Multi-robot cooperation using the ant algorithm with variable pheromone placement," in Evolutionary Computation, 2005. The 2005 IEEE Congress on, vol. 2, 2005, pp. 1232 1237 Vol. 2.

[13] D. Zhang, G. Xie, J. Yu, and L. Wang, "An adaptive task assignment method for multiple mobile robots via swarm intelligence approach," in Computational Intelligence in Robotics and Automation, 2005. CIRA 2005. Proceedings. 2005 IEEE International Symposium on, 2005, pp. $415-420$.

[14] K. Ohkura, T. Yasuda, and Y. Matsumura, "Coordinating the adaptive behavior for swarm robotic systems by using topology and weight evolving artificial neural networks," in Evolutionary Computation (CEC), 2010 IEEE Congress on, 2010, pp. $1-8$.

[15] L. Fan and P. Dasgupta, "A Stigmergy-Based Model for Solving Cooperative Pursuit-Evasion Games in Unknown Environments," in SASO '08: Proceedings of the 2008 Second IEEE International Conference on Self-Adaptive and Self-Organizing Systems. Washington, DC, USA: IEEE Computer Society, 2008, pp. 467-468.

[16] A. Purnamadjaja and R. Russell, "Pheromone communication: implementation of necrophoric bee behaviour in a robot swarm," in Robotics, Automation and Mechatronics, 2004 IEEE Conference on, vol. 2, 2004, pp. $638-643$ vol.2.

[17] - "Robotic pheromones: Using temperature modulation in tin oxide gas sensor to differentiate swarm's behaviours," in Control, Automation, Robotics and Vision, 2006. ICARCV '06. 9th International Conference on, 2006, pp. $1-6$.

[18] A. Chatty, I. Kallel, and A. M. Alimi, "Counter-ant algorithm for evolving multirobot collaboration," in Proceedings of the 5th international conference on Soft computing as transdisciplinary science and technology, ser. CSTST '08. ACM, 2008, pp. 84-89.

[19] I. Kallel, A. Chatty, and A. Alimi, "Self-organizing multirobot exploration through counter-ant algorithm," in Self-Organizing Systems, ser. Lecture Notes in Computer Science, K. Hummel and J. Sterbenz, Eds. Springer Berlin / Heidelberg, 2008, vol. 5343, pp. 133-144.

[20] A. Chatty, I. Kallel, and A. M. Alimi, "Fuzzy counter-ant for avoiding the stagnation ofmultirobot exploration," in Proceedings of the IEEE Int. Conf on Systems, Man, and Cybernetics SMC, ser. SMC, 2010, pp. 140-148.

[21] M. Mataric, "Designing emergent behaviors ; from local interactions to collective intelligence," From animals to animats, pp. 1-6, 1992.

[22] E. Bonabeau, G. Theraulaz, V. Fourcassié, and J.-L. Deneubourg, "Phase-ordering kinetics of cemetery organization in ants," Phys. Rev. E, vol. 57, no. 4, pp. 4568-4571, Apr 1998.

[23] P. Gaussier and S. Zrehen, "Avoiding the world model trap: An acting robot does not need to be so smart!" Robotics and Computer-Integrated Manufacturing, vol. 11, no. 4, pp. 279 - 286, 1994.

[24] R. Beckers, O. E. Holl, and J. L. Deneubourg, "From local actions to global tasks: Stigmergy and collective robotics," in In R. Brooks and P. Maes, editors, Proceedings of the Fourth Workshop on Artificial Life. Cambridge, MA: MIT Press, 1994, pp. 181-189.
[25] A. Martinoli and F. Mondada, "Collective and cooperative group behaviours: Biologically inspired experiments in robotics," in Experimental Robotics IV, ser. Lecture Notes in Control and Information Sciences, O. Khatib and J. Salisbury, Eds. Springer Berlin / Heidelberg, 1997, vol. 223, pp. 1-10.

[26] T. Lan, S. Liu, and S. Yang, "Collective cooperation inspired by stigmergy strategy," in Intelligent Control and Automation, 2006. WCICA 2006. The Sixth World Congress on, 02006.

[27] M. Martin, B. Chopard, and P. Albuquerque, "Formation of an ant cemetery: swarm intelligence or statistical accident?" Future Generation Computer Systems, vol. 18, no. 7, pp. 951 - 959, 2002.

[28] C. Kube and H. Zhang, "Stagnation recovery behaviours for collective robotics," in Intelligent Robots and Systems '94. 'Advanced Robotic Systems and the Real World', IROS '94. Proceedings of the IEEE/RSJ/GI International Conference on, vol. 3, Sep. 1994, pp. 1883 -1890 vol.3.

[29] P. Laroque, N. Gaussier, N. Cuperlier, M. Quoy, and P. Gaussier, "Cognitive map plasticity and imitation strategies to improve individual and social behaviors of autonomous agents," Paladyn, vol. 1, pp. 25-36, 2010.

[30] J. O'Keefe and L. Nadel, The hippocampus as a cognitive map. Clarendon Press ; Oxford University Press, December 1978.

[31] P. Gaussier, A. Revel, J. P. Banquet, and V. Babeau, "From view cells and place cells to cognitive map learning: processing stages of the hippocampal system," Biological Cybernetics, vol. 86, pp. 15-28, 2002. 